\title{
Valoración de un diseño educativo tecnológico para prevenir el abandono escolar
}

\author{
Ma Victoria Martín-Cilleros ${ }^{1}$, Andrés Sánchez-Prada², María Carmen Delgado-Álvarez ${ }^{2}$ \\ y Ma Cruz Sánchez-Gómez ${ }^{1}$
}

viquimc@usal.es, asanchezpr@upsa.es, mcsago@usal.es

${ }^{1}$ Departamento de Didáctica, Organización y Métodos de Investigación Universidad Salamanca, Spain

${ }^{2}$ Universidad Pontificia de Salamanca, Spain

DOI: 10.17013/risti.23.61-77

\begin{abstract}
Resumen: Se presentan resultados de una investigación subvencionada por el Ministerio de Educación Pública de Costa Rica y la Unión Europea, cuya finalidad es dar respuesta al problema del abandono escolar a través de un proyecto implementado fuera del horario escolar. Supone una gestión educativa participativa, donde los estudiantes se sientan protagonistas de los procesos formativos y se fortalezca su sentido de pertenencia a la comunidad, a través de actividades innovadoras como son los medios audiovisuales. Una visión global para diagnosticar el estado de la cuestión aconsejó utilizar el enfoque cuantitativo y una profundización comprensiva e interpretativa mayor de las dimensiones a estudiar, advirtió la utilización del cualitativo. Para la obtención de los datos se optó por un cuestionario semiestructurado y cumplimentado por 170 estudiantes. Los resultados indican la multiplicidad de factores de riesgo en el absentismo y abandono escolar que exigen intervenciones igualmente múltiples de las diferentes administraciones e instituciones.
\end{abstract}

Palabras clave: deserción escolar; absentismo; medios audiovisuales; proyecto extracurricular; enfoque multimétodo.

\section{Assessment of a technological education design to prevent school dropout}

Abstract: The article reports the findings of a research project funded by the Ministry of Public Education of Costa Rica and the European Union. The purpose is to provide an answer to the problem of school dropout through a project implemented outside school hours. It involves a participatory approach to education where students can feel protagonists of the training process and strengthen their sense of belonging to a community through innovative activities including audiovisual media. A global assessment to diagnose the status of the matter revealed the suitability of a quantitative approach, and further understanding and interpretation of the dimensions to be studied suggested the use of a qualitative approach. Data were gathered using a semi-structured questionnaire drawn up ad hoc and filled out by 170 students. The results reveal the numerous risk factors involved in school absenteeism and dropout, which in turn require equally multiple types of interventions by the different administrations and institutions. 
Keywords: school drop out; absenteeism; audiovisual media; extracurricular project; multimethod approach.

\section{Introducción}

Los estudios sobre el absentismo identifican múltiples causas del mismo. Así en el programa elaborado por el Gobierno Vasco (2003) establecen diferentes causas: causas centradas en el entorno, en la familia, en el propio estudiante y otras causas que complementan a las anteriores y en ocasiones las agravan como son las razones de género y culturales.

Entre las causas centradas en el entorno, y siguiendo el documento citado anteriormente, se encuentran las normas y valores del grupo de iguales que pueden influir en el estudiante, así como un entorno social deprimido. En relación a las causas centradas en la escuela, se incluyen los métodos utilizados, individualistas y competitivos, la falta de integración en el barrio o en el municipio, un proyecto pedagógico no consensuado, donde no hay una labor de equipo entre los docentes, el profesorado inestable en el centro, problemas de adaptación de los alumnos a la escuela, el clima de convivencia y de práctica del estudio, o el ambiente percibido en las clases y fuera de ellas. Un contexto familiar con problemas económicos, o relaciones conflictivas en la familia, con problemas de toxicomanías o asociados como la prostitución, la situación de prisión, son factores igualmente relacionados con el absentismo. Entre las causas centradas en la o el joven se pueden encontrar con factores como la baja autoestima, el sentimiento de falta de competencia, el sentirse desplazado o mal considerado, los trastornos psicológicos/psiquiátricos, la mala preparación académica anterior, los malos resultados académicos, una salud física delicada, el bajo nivel de sintonía con el profesorado y con el propio tutor o tutora, la respuesta a la norma establecida (en preadolescentes y adolescentes), la predelincuencia infanto-juvenil o los trastornos de conducta del menor o la menor en la familia.

En algunos países lationoamericanos, todas estas causas convergen siendo todo un reto para las políticas del país enfrentarse al absentismo escolar. Tal es la situación de Costa Rica, país que ha logrado una cobertura educacional cercana al 100\%, pero con una gran problemática del abandono escolar en educación secundaria. Para enfrentar dichas dificultades, el Gobierno ha desarrollado esfuerzos por expandir el servicio educativo y mejorar su calidad en todos los niveles y modalidades, introduciendo modificaciones curriculares que le den valor agregado a los planes de estudio, creando alternativas para quienes no asisten a la educación formal, y proporcionando becas, bonos escolares, transporte y alimentación para que los alumnos permanezcan escolarizados (Arguedas \& Jiménez, 2007).

Todas estas acciones han resultado significativas, pero no han sido suficientes para reducir sustancialmente el abandono escolar, especialmente en determinados centros educativos por encontrarse éstos insertos en zonas con alta vulnerabilidad y situación de pobreza. En este escenario, uno de los factores asociados al abandono escolar es el escaso interés y motivación de los alumnos por los estudios, lo cual se deriva de modalidades pedagógicas poco atractivas y que no logran despertar su creatividad, así como una educación poco pertinente, con contenidos no significativos y alejados de la realidad e intereses de los estudiantes. A esto, se suma la sensación de que los profesores no conocen la realidad comunal y familiar en la que están insertos, especialmente, cuando se trata de contextos socioeconómicos deprimidos. Todo lo anterior tiene como primera consecuencia el 
ausentismo escolar, posteriormente, el bajo rendimiento, y al final, la deserción (Jiménez \& Gaete, 2010; Abreu et al., 2017). A su vez, existen elementos que contribuyen a la permanencia de los jóvenes en las instituciones educativas. Dentro de ellos, se encuentra el énfasis en una formación integral, que vaya más allá de lo estrictamente académico; las experiencias de éxito; la construcción de un sentido de pertenencia a la comunidad educativa, con redes de apoyo significativas, tanto entre los docentes, como en el grupo de pares; así como el uso de metodologías educativas que generen interés, con contenidos relevantes para los adolescentes, para sus vidas presentes y futuras, lo cual genere una motivación intrínseca hacia el estudio (Arguedas, 2011).

Tanto los factores asociados al abandono escolar como los que promueven la permanencia en él, fueron tenidos en cuenta para la elaboración de un programa contra el absentismo escolar. Partiendo de la consideración de la realidad de cada establecimiento educacional, se busca implementar una propuesta significativa y estimulante para los estudiantes, que los conecte con los aspectos positivos de las comunidades en las que viven y les permita aprender y experimentar con un medio de comunicación atractivo como es el audiovisual.

\section{Descripción del proyecto.}

El programa se enmarca dentro de un convenio internacional entre el Ministerio de Educación Pública de Costa Rica y la Unión Europea. A través de un proyecto extracurricular se pretende desarrollar una gestión educativa participativa y dar respuesta al problema de la deserción escolar, lograr que los estudiantes encuentren los procesos educativos interesantes y que se fortalezca su sentido de pertenencia a la comunidad a través de actividades innovadoras y diferentes, en el cual los adolescentes deben ser los protagonistas y desarrollar su creatividad. Para ello se ha utilizado instrumentos tecnológicos atractivos, como son los medios audiovisuales.

El que se trate de un proyecto extracurricular contribuirá a liberarlo de las asociaciones que los estudiantes tienen con los deberes, calificaciones, asignaturas, etc., fomentando el desarrollo de una motivación intrínseca ante situaciones de aprendizaje no formal. Asimismo, se favorecerá la formación integral de los estudiantes, considerando elementos académicos (capacidades de expresión y comunicación, conocimiento del entorno, historia, etc.), junto a factores psicosociales como son el ámbito emocional (autoestima, autoeficacia, construcción de la propia identidad, entre otros) y de relaciones interpersonales (por ejemplo, mediante el trabajo en equipo, que promueva las relaciones entre los alumnos y genere redes de apoyo sólidas dentro de la escuela).

En el desarrollo del proyecto se definieron diferentes fases:

Fase 1: Capacitación y formulación del proyecto adaptado a las características particulares de cada establecimiento educacional. Esta fase consiste en la capacitación de los actores relevantes, tanto del diseño e implementación de proyectos extracurriculares, como en lo concerniente a temas técnicos vinculados a la producción audiovisual.

Fase 2: Implementación del programa extracurricular en cada comunidad. Dicho programa se desarrolló mediante talleres, orientados a la elaboración de documentales que vinculen a los estudiantes a sus contextos y realidades. 
Fase 3: Difusión de resultados. Con los micro-documentales terminados, se procedería a realizar un evento comunal oficial donde se presentan los resultados.

\section{Metodología}

\subsection{Participantes.}

Los participantes provienen de tres centros educativos diferentes, enclavados en zonas con índices bajos de desarrollo humano, en relación con el resto del país. En ellos se destaca la diversidad cultural, con presencia de población indígena, afrodescendientes e inmigrantes, un alto índice de analfabetismo y baja tasa de matriculación secundaria.

Aunque el proyecto iba dirigido a todos los estudiantes de los centros, finalmente en su evaluación se ha contado con las respuestas de 82 alumnos y 88 alumnas para la evaluación de los resultados, un total de 170 discentes.

\subsection{Preguntas de la investigación y objetivos.}

El proyecto se cuestiona si a través de las tecnologías se potencia la motivación del alumnado, si además en lugar de incluirlas en el curriculum oficial, un programa extracurricular es pertinente para vincular a los estudiantes al centro educativo y, si la actividad propuesta adicionalmente ayuda a fomentar un sentimiento de pertenencia a la comunidad. Ante estas preguntas se plantea como objetivo general fomentar en el alumnado la permanencia en el ámbito escolar a través de un Proyecto Extracurricular orientado a la realización de microdocumentales sobre aspectos significativos de la comunidad de referencia. Para ello se plantean los siguientes objetivos específicos: a) fomentar en el alumnado la motivación para permanecer en el centro educativo, mediante la experiencia en actividades creativas y de cohesión grupal, que refuercen el vínculo entre la comunidad y el centro escolar y b) potenciar entre los jóvenes habilidades sociales y competencias que puedan tener una proyección de futuro hacia el ámbito laboral, dando sentido a la formación en el centro escolar

\subsection{Método}

La evaluación de este proyecto, su objetivo general, las dimensiones que se quieren analizar y su objeto de estudio obligaban a emplear una estrategia metodológica multimétodo, que convenga en un compromiso entre las orientaciones cuantitativa y cualitativa de la investigación social. No todas las observaciones son susceptibles de medición cuantitativa, más aún cuando se trabaja sobre la escurridiza cuestión de las preferencias e intereses de los actores y la captación de sus discursos. Además, la comparación obliga a establecer diferenciaciones no sólo en términos de cantidad (cuantitativas) sino de cualidad (cualitativas) (Ragin, 2014).

En relación al diseño cuantitativo, se utilizó la metodología cuasiexperimental porque es la más indicada cuando la investigación cuantitativa se desarrolla en escenarios educativos naturales. La posibilidad de una profundización comprensiva e interpretativa mayor de las dimensiones a estudiar, así como su análisis específico y pormenorizado, aconseja también la utilización del enfoque cualitativo. Aunque exigen la concentración 
en ámbitos delimitados contextualmente y no permiten una universalización o extensión generalizada -para eso utilizaremos la investigación cuantitativa-, hacen posible un estudio más detallado. Y ello es posible, en primer lugar, porque la utilización de métodos cualitativos tiene como característica principal el análisis y la interpretación del significado que las personas dan a sus acciones y a las acciones de los demás y, en segundo término, porque el uso de métodos cualitativos requiere del trato directo, en los contextos particulares de relación y de diálogo, con las personas que interactúan en los entornos concretos y participan en y de los procesos que se pretenden analizar (Gómez, Vicario, García \& García 2014). En un primer momento, se pensó establecer grupos focales recurriendo además a información procedente de otras fuentes, como: documentos oficiales, anecdotarios, etc. Sin embargo, con el fin de facilitar la recogida de información de todos los datos, se optó por no realizar dichas entrevistas grupales puesto que los centros estaban dispersos geográficamente y era difícil que estudiantes y profesores se desplazaran para la ejecución de los mismos. Por ello se añadieron respuestas abiertas al cuestionario elaborado, con el mismo objetivo inicial de conocer el discurso, concepciones, expectativas, prácticas y dificultades de los participantes en la acción educativa. Mediante las opiniones expresadas tanto en los cuestionarios como posteriormente en entrevistas realizadas por un observador externo en el propio contexto tanto a directores/as, como coordinadores/as del proyecto, profesorado y alumnado participante, se recogen las diferentes causas del entorno, familiares, escolares, personales y de algún otro tipo como culturales, que pueden estar influyendo en el discente. Se trata de conocer el estado actual, cuáles son las debilidades y fortalezas.

\subsection{Instrumentos de evaluación}

Se elaboró ad hoc un cuestionario, dirigido a los estudiantes, cuyo contenido general se puede agrupar en bloques: a) datos personales, familiares y socioculturales, b) pruebas para medir actitudes de los participantes hacia la escuela, compañeros, etc. y c) preguntas abiertas para conocer las opiniones de los participantes sobre diferentes aspectos que influyen en la asistencia escolar. Para estos dos últimos aspectos se realizó una adaptación del "Phycological Sense of School Membership" (PSSM) de Goodenow (1993) utilizado posteriormente por Ros (2014) para analizar los factores que afectan al sentimiento de pertenencia como aspecto clave para evitar las altas tasas de abandono y fracaso escolar de los estudiantes.

\subsection{Procedimiento}

El proceso de evaluación se llevó a cabo al inicio del proyecto, antes incluso de la capacitación inicial, para establecer la línea base y poder contar con indicadores para medir el impacto del proyecto en términos de satisfacción y conocimientos adquiridos. Esta evaluación se extendió hasta el final de la experiencia, utilizando cuestionarios diseñados inicialmente y adaptados a un formato electrónico con el fin de evitar material fungible. En la fase final se realizaron entrevistas en el propio contexto tanto a directores/ as, como coordinadores/as del proyecto, profesorado y alumnado participante.

Los datos de carácter cuantitativo se analizaron, desde perspectivas descriptivas e inferenciales, con la aplicación del programa SPSS, ajustando en todos los casos los tipos de análisis a la naturaleza métrica o no métrica de las variables tratadas. En los análisis 
descriptivos, por ejemplo, se utilizaron técnicas relacionales específicas, en función del tipo de variables: tablas de contingencia e índices de correlación tau propio de variables no cuantitativas; medidas de tendencia central y variabilidad.

En lo que respecta a los análisis inferenciales se utilizaron técnicas de análisis multivariantes que permitan captar la complejidad de las interrelaciones e interacciones entre las variables con el fin de explicar del modo más comprehensivo y parsimonioso posible, la eficacia del programa. Se utilizaron igualmente técnicas de dependencia para analizar el efecto de las variables independientes sobre las dependientes (MANOVA).

En el análisis de carácter cualitativo el programa NVIVO11 es muy útil en la fase de obtención de resultados y verificación de conclusiones ya que permite usar herramientas de análisis de texto para extraer los resultados exploratorios que permitirán avanzar en la explicación, comprensión y conocimiento de la realidad, y contribuir a la teorización o intervención sobre la misma (Sánchez-Gómez y Martín-Cilleros, 2017).

\section{Resultados}

Se obtuvieron respuestas válidas de un total de 170 participantes, 82 alumnos $(48,2 \%)$ y 88 alumnas $(51,8 \%)$ de los tres centros que finalmente formaron parte del proyecto. Del total de participantes en esta fase del proyecto, $106(62,4 \%)$ estudiaban en el LS, 57 (33,5 $\%$ ) en el RC, y siete $(4,1 \%)$ en el IP. El porcentaje de alumnos y alumnas provenientes de cada uno de los tres centros resultó equivalente en términos estadísticos (Coeficiente de Contingencia $=0,041 ; \mathrm{p}=.866)$, tal y como se puede apreciar en la tabla 1 .

\begin{tabular}{llllllll}
\hline SEXO / CENTRO & \multicolumn{1}{l}{ RC } & \multicolumn{2}{l}{ LS } & IP & Total \\
\hline Alumnas & 28 & $49,1 \%$ & 56 & $52,8 \%$ & 4 & $57,1 \%$ & 88 \\
\hline Alumnos & 29 & $50,9 \%$ & 50 & $47,2 \%$ & 3 & $42,9 \%$ & 82 \\
\hline TOTAL & 57 & $100 \%$ & 106 & $100 \%$ & 7 & $100 \%$ & 170 \\
\hline
\end{tabular}

\begin{tabular}{llllllll}
\hline CURSO / CENTRO & \multicolumn{1}{l}{ RC } & \multicolumn{2}{l}{ LS } & IP & Total \\
\hline $1^{\mathrm{o}}$ curso & 34 & $59,6 \%$ & 33 & $31,1 \%$ & --- & 67 \\
\hline $2^{\mathrm{o}}$ curso & -- & & 29 & $27,4 \%$ & --- & 29 \\
\hline $3^{\mathrm{o}}$ curso & 16 & $28,1 \%$ & 14 & $13,2 \%$ & 7 & $100 \%$ & 37 \\
\hline $4^{\mathrm{o}}$ curso & 7 & $12,3 \%$ & 13 & $12,3 \%$ & --- & & 20 \\
\hline $5^{\mathrm{o}}$ curso & --- & & 15 & $14,1 \%$ & --- & 15 \\
\hline $6^{\mathrm{o}}$ curso & --- & & 2 & $1,9 \%$ & --- & 2 \\
\hline Total & 57 & $100 \%$ & 106 & $100 \%$ & 7 & $100 \%$ & 170 \\
\hline
\end{tabular}




\begin{tabular}{lccccl}
\hline CURSO / SEXO & \multicolumn{2}{c}{ Alumnos } & \multicolumn{1}{c}{ Alumnas } & Total \\
\hline $1^{\circ}$ curso & 26 & $31,7 \%$ & 41 & $46,6 \%$ & 67 \\
\hline $2^{\circ}$ curso & 16 & $19,6 \%$ & 13 & $14,8 \%$ & 29 \\
\hline $3^{\circ}$ curso & 17 & $20,7 \%$ & 20 & $22,7 \%$ & 37 \\
\hline $4^{\circ}$ curso & 14 & $17,1 \%$ & 6 & $6,8 \%$ & 20 \\
\hline $5^{\circ}$ curso & 7 & $8,5 \%$ & 8 & $9,1 \%$ & 15 \\
\hline $6^{\circ}$ curso & 2 & $2,4 \%$ & --- & & 2 \\
\hline Total & 82 & $100 \%$ & 88 & $100 \%$ & 170 \\
\hline
\end{tabular}

Tabla 1 - Distribución por sexo/centro; curso/centro; sexo/curso de la muestra

Respecto al nivel educativo cursado, los/as participantes se distribuían del modo siguiente: $67(39,4 \%)$ en primero, 29 (17\%) en segundo, $37(21,8 \%)$ en tercero, 20 $(11,8 \%)$ en cuarto, $15(8,8 \%)$ en quinto, y dos $(1,2 \%)$ en sexto curso. El tiempo promedio de permanencia en sus respectivos centros en el momento de la evaluación era de $\mathrm{M}=$ 2,28 años (DT = 1,55), sin diferencias significativas entre sexos $(\mathrm{t} 164=1,206 ; \mathrm{p}=.230)$.

Finalmente, en la tabla 1 se muestran las distribuciones por curso, que fueron significativamente diferentes entre los centros (Coeficiente de Contingencia= 0,521; $\mathrm{p}<.001)$. El centro RC aportó alumnado de $1^{\circ}, 3^{\circ}$ y $4^{\circ}$ curso, al igual que el centro LS, que aportó además alumnado de $2^{\circ}, 5^{\circ}$ y $6^{\circ}$ (de éste último, únicamente dos participantes). Los siete estudiantes provenientes del Centro IP pertenecían al $3^{\circ}$ curso. Por otra parte,

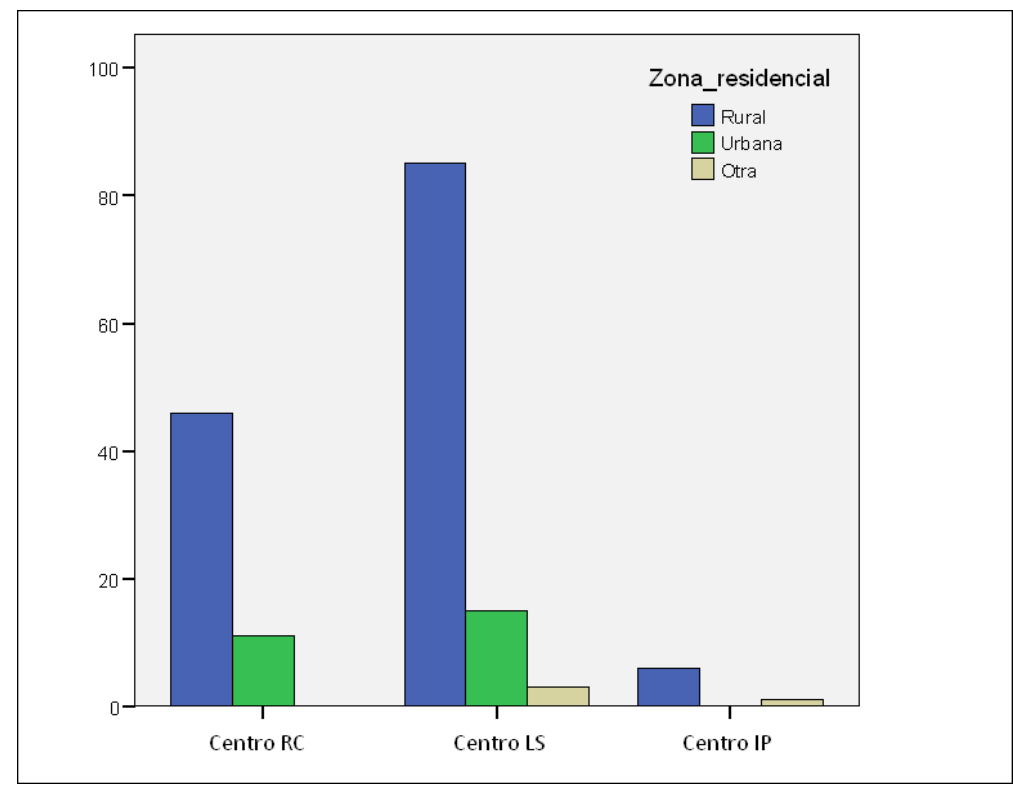

Figura 1 - Área de residencia según centro 
a pesar de las aparentes diferencias que se pueden observar, no hay relación significativa entre el nivel educativo cursado y el sexo del alumnado $(\mathrm{p}=.110)$.

En cuanto al área residencial, del total de participantes $137(80,6 \%)$ vivían en zonas rurales, frente $26(15,3 \%)$ afincados/as en zonas urbanas. Cuatro sujetos (2,3\%) señalaron que su zona de residencia era "otra", y de los restantes tres (1,8\%) no se obtuvo respuesta. Como se refleja en la figura 1, la participación alumnado proveniente de la zona rural fue mayoritaria en los tres centros, en proporciones equivalentes: 46 casos de 57 (80,7\%) en el RC, 85 de 106 (82,5\%) en el LS, y seis de siete (85,7\%) en el IP (Coeficiente de Contingencia $=0,205 ; \mathrm{p}=.121$ ).

La tabla 2 muestra la distribución del alumnado según sexo en cada zona de residencia. A pesar de las diferencias, en términos absolutos, en el número de participantes, los porcentajes de alumnas y alumnos pueden ser considerados estadísticamente equivalentes a través de las tres zonas (Coeficiente de Contingencia= 0,123; $\mathrm{p}=.278$ ).

\begin{tabular}{llllll}
\hline ZONA / SEXO & \multicolumn{1}{c}{ Alumnas } & \multicolumn{1}{c}{ Alumnos } & Total \\
\hline Rural & 75 & $86,2 \%$ & 62 & $77,5 \%$ & 137 \\
\hline Urbana & 11 & $12,7 \%$ & 15 & $18,7 \%$ & 26 \\
\hline Otra & 1 & $1,1 \%$ & 3 & $3,8 \%$ & 4 \\
\hline Total & 87 & $100 \%$ & 80 & $100 \%$ & 167 \\
\hline
\end{tabular}

Tabla 2 - Distribución área de residencia/sexo de los participantes

En lo que respecta a la contribución económica a la unidad familiar, en número de personas que aportan algún tipo de ingreso osciló, a tenor de las respuestas obtenidas, entre cero y nueve $(M=1,62 ; \mathrm{DT}=0,93)$. Se observó una ligera diferencia entre las zonas rurales y las urbanas en este sentido $(M=1,65$ y $M=1,31$, respectivamente), si bien resultó nula en términos estadísticos $(\mathrm{t}(160)=1,742 ; \mathrm{p}=.083)$.

Por otra parte, aproximadamente la mitad de la muestra tiene progenitores con estudios primarios cursados (entre un $44 \%$ y un $45 \%$; véase tabla 3), y en torno a una quinta parte

\begin{tabular}{lllll}
\hline NIVEL DE ESTUDIOS & Padre & \multicolumn{2}{c}{ Madre } \\
\hline Sin estudios & 42 & $24,7 \%$ & 33 & $19,4 \%$ \\
\hline Primarios & 76 & $44,7 \%$ & 75 & $44,1 \%$ \\
\hline Secundarios & 32 & $18,8 \%$ & 37 & $21,8 \%$ \\
\hline Bachiller & 4 & $2,4 \%$ & 15 & $8,8 \%$ \\
\hline Formación profesional & 9 & $5,3 \%$ & 7 & $4,1 \%$ \\
\hline Superiores & 2 & $1,2 \%$ & --- & $-1,8 \%$ \\
\hline NS/NC & 5 & $2,9 \%$ & 3 & $100 \%$ \\
\hline Total & 170 & $100 \%$ & 170 & 100 \\
\hline
\end{tabular}

Tabla 3 - Estudios de los progenitores o tutores 
tiene estudios secundarios (18,8\% de los padres y 21,8\% de las madres). Los porcentajes resultan similares en los casos de madres y padres sin estudios (24,7\% de los padres y un 19,4\% de las madres). El porcentaje de madres con estudios de bachiller es notablemente superior $(8,8 \%)$ al de padres $(2,4 \%)$; en cambio, únicamente 2 progenitores han cursado estudios superiores, en ambos casos padres (1,2\%).

La edad promedio de los progenitores fue de 38,3 años en el caso de las madres (DT=6,8; rango entre 25 y 56), y de 43,6 años en los padres (DT= 7,9; rango entre 15 y 69), y en su mayoría estaban casadas/os o vivían juntos (48,8\%). Finalmente, en los casos en que se convivía con hermanos/as en casa, su número osciló entre uno y 15 ( $\mathrm{M}=3,06$; DT= 2,07), siendo lo más frecuente un número de hermanos entre uno y cinco ( $91 \%$ de los casos).

Entrando en las variables más directamente relacionadas con la auto-percepción del alumnado respecto al centro educativo, en la tabla 4 se muestran los datos correspondientes al sentimiento de pertenencia al centro, posiblemente un elemento clave en relación al abandono y al fracaso escolar. En términos generales se aprecia una buena valoración de esta dimensión, con puntuaciones medias en todos los ítems superiores a 3,5 en una escala de 5 puntos.

\begin{tabular}{ll}
\hline SENTIMIENTO DE PERTENENCIA & Media \\
\hline Creo que mi Centro es un buen colegio & 3,96 \\
\hline Me siento parte de este colegio & 4,09 \\
\hline Se puede decir que en general disfruto en mi colegio & 4,02 \\
\hline Me siento “como en casa” en este colegio & 3,60 \\
\hline Hablo con frecuencia bien del colegio con mi familia amigos o conocidos & 3,94 \\
\hline Recomendaría este colegio a otros amigos, familiares... & 3,97 \\
\hline Estoy orgulloso de mi colegio & 3,95 \\
\hline
\end{tabular}

Tabla 4 - Medias obtenidas en la dimensión sentimiento de pertenencia

En el mismo sentido se manifiestan los descriptivos de la dimensión sentimiento de grupo y participación, cuyos ítems recibieron buenas valoraciones (medias superiores a 3,9 en una escala de 5 puntos; véase tabla 5).

\begin{tabular}{ll}
\hline GRUPO Y PARTICIPACIÓN & Media \\
\hline Los diferentes miembros de este colegio tenemos espíritu de equipo & 3,96 \\
\hline En nuestra clase estamos muy unidos & 4,09 \\
\hline En el colegio se fomenta mi participación & 4,02 \\
\hline
\end{tabular}

Tabla 5 - Medias obtenidas en la dimensión sentimiento de grupo y participación 
Asimismo, como se observa en la tabla 6, el alumnado valora positivamente todos los ítems relacionados con el estudio y la percepción que tienen de sí mismos al respecto, con medias en todos los ítems superiores a 3,5 en una escala de 5 puntos.

\begin{tabular}{ll}
\hline VALORACIÓN DEL ESTUDIO & Media \\
\hline Sé que seré capaz de aprobar este curso & 4,45 \\
\hline Considero que estoy aprendiendo mucho & 4,28 \\
\hline Estoy contento con mis notas & 3,59 \\
\hline Creo que soy un buen estudiante & 3,94 \\
\hline Me gusta estudiar & 3,79 \\
\hline Las buenas notas son muy importantes para mí & 4,56 \\
\hline Espero con ilusión cada nuevo curso escolar & 4,18 \\
\hline
\end{tabular}

Tabla 6 - Medias obtenidas en la dimensión valoración del estudio

Por otra parte, se aprecia una buena relación con el profesorado, con valoraciones positivas de su implicación y desempeño (medias en todos los ítems superiores a 3,6 en una escala de 5 puntos; véase la tabla 7).

\begin{tabular}{ll}
\hline RELACIÓN CON PROFESORADO & Media \\
\hline Los profesores/as se preocupan por mis problemas & 3,72 \\
\hline Los profesores/as toman mis opiniones en serio y me entienden & 3,93 \\
\hline Los profesores/as me ayudan cuando lo necesito & 3,97 \\
\hline En clase me siento cómodo/a con casi todos los profesores/as & 4,09 \\
\hline Los profesores/as me prestan atención fuera de su tiempo de clase & 3,82 \\
\hline En general tengo buena relación con todos los profesores/as & 4,01 \\
\hline Hay al menos un maestro u otro adulto en esta escuela con el que siento bien & 3,67 \\
\hline Los profesores son justos, me elogian si he hecho bien algo & 4,19 \\
\hline
\end{tabular}

Tabla 7 - Medias obtenidas en la dimensión relación con el profesorado

Para finalizar el análisis descriptivo de las dimensiones, en la tabla 8 se puede apreciar la actitud positiva que el alumnado percibe en su familia respecto al centro y a sus estudios: en una escala de 5 puntos, las medias en todos los ítems directos son superiores a 3,7 (excepto en lo relativo a la participación en las actividades, con puntuaciones relativamente inferiores); y las medias en los ítems inversos son iguales o inferiores a 2 puntos. 


\begin{tabular}{ll}
\hline ACTITUD FAMILIAR & Media \\
\hline Mis padres me preguntan sobre lo que hacemos en clase & 4,13 \\
\hline Mis padres me sirven de ayuda al hacer los deberes & 4,08 \\
\hline A mis padres les da igual si vengo o no al colegio & 2,01 \\
\hline Mi familia no me deja venir al colegio & 1,82 \\
\hline Creo que mis estudios y estar en este colegio son muy importante & 4,53 \\
\hline Mi familia me anima a venir al colegio & 4,50 \\
\hline Mis padres participan mucho en la vida del colegio y se implican & 3,76 \\
\hline Mis padres están contentos con el colegio & 4,26 \\
\hline Mis padres participan en actividades del colegio & 2,89 \\
\hline Mi casa es un lugar adecuado para poder estudiar y hacer labores & 4,14
\end{tabular}

Tabla 8 - Medias obtenidas en la dimensión actitud familiar

Se analizaron las posibles diferencias entre sexos para cada uno de los ítems evaluados mediante MANOVA. En la mayoría de los ítems las diferencias observadas fueron estadísticamente nulas; únicamente se aprecian diferencias significativas en los ítems que se muestran a continuación (tabla 9).

\begin{tabular}{llll}
\hline DIFERENCIAS POR SEXO & Niños & Niñas & p \\
\hline Sé que seré capaz de aprobar este curso & 4.30 & 4.59 & 0.043 \\
\hline Participo en campamentos de verano, salidas, excursiones... & 2.79 & 2.27 & 0.031 \\
\hline Cuando tengo alguna dificultad mis compañeros/as me ayudan & 3.71 & 4.14 & 0.018 \\
\hline Tengo buenos amigos/as entre mis compañeros/as de clase & 4.06 & 4.39 & 0.044 \\
\hline Tengo más amigos/as de mi zona de residencia que en clase & 3.76 & 3.32 & \multirow{2}{*}{0.034} \\
\hline Mis amigos/as me influyen positivamente en el estudio & 3.95 & 4.36 & \multirow{2}{*}{0.021} \\
\hline
\end{tabular}

Tabla 9 - Diferencias por sexo

A partir de las comparaciones ítem a ítem, se podría afirmar que las alumnas muestran una mayor confianza en su capacidad para superar el curso que los alumnos, y éstos participan más en campamentos, salidas o excursiones. En los ítems restantes, sobre aspectos de relación entre pares, se observa que las alumnas tienden a establecer más relaciones de amistad en el centro, en comparación con los alumnos, y éstos tienden a tener más amistades en su zona de residencia.

Por otra parte, el análisis con MANOVA de las posibles diferencias entre centros arrojó diferencias significativas en nueve ítems, que se muestran en la figura 2. 


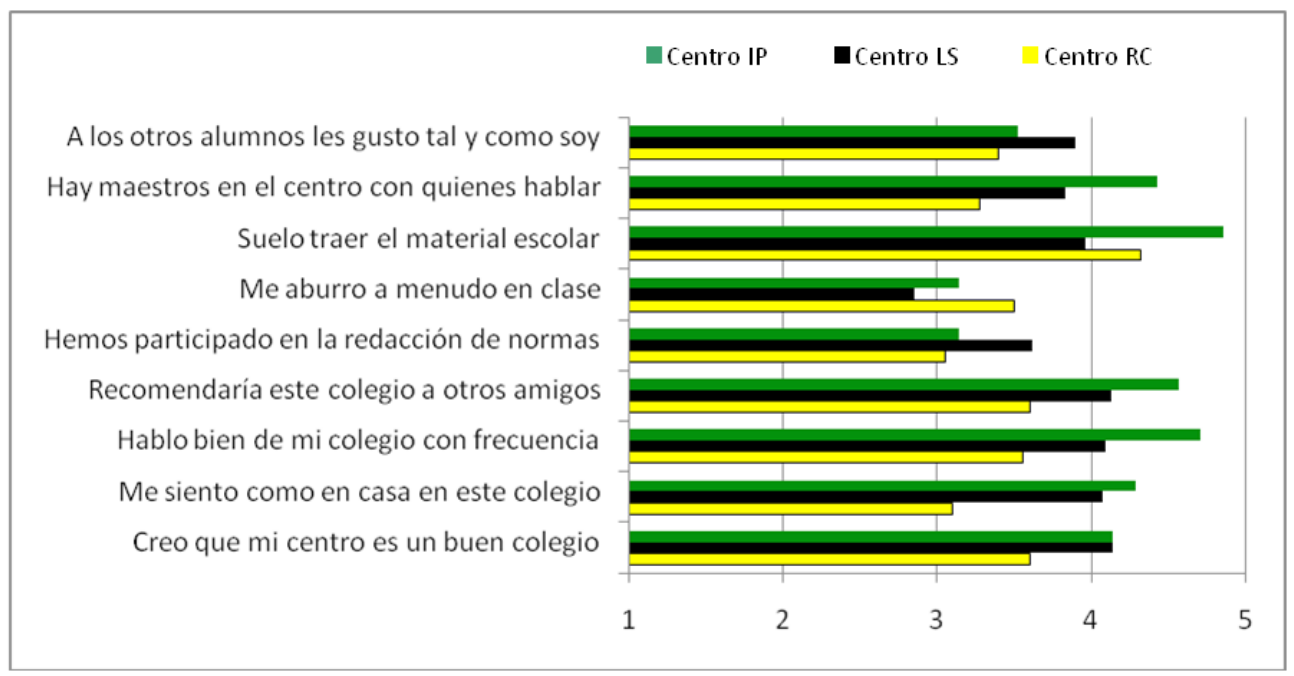

Figura 2 - Comparación de medias por centro

A fin de recabar indicios que permitieran valorar el impacto del programa formativo, se elaboró un cuestionario dirigido a conocer el grado de capacitación auto-percibida por los/as participantes, y la valoración que éstos/as hacen del programa y de su utilidad.

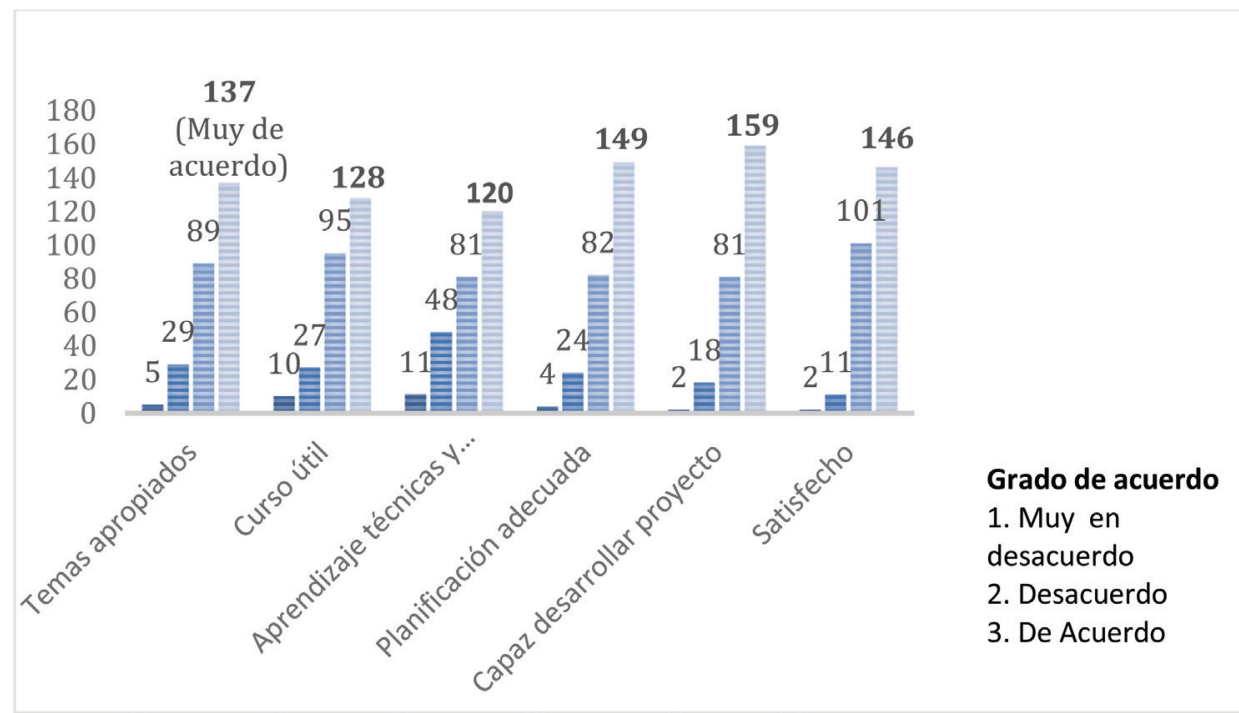

Figura 3 - Valoración del programa (frecuencias absolutas)

Como se observa en la figura 3, la formación recibida ha hecho que los participantes se sintieran capaces de desarrollar el proyecto, aprendiendo diferentes técnicas y estrategias para desarrollarlo. Muchas de estas técnicas conllevan trabajar en equipo, lo que permite la adquisición de competencias transversales importantes para un futuro. 
El 94,65\% de los participantes que respondieron al cuestionario se sentían satisfechos (puntuaciones 3-4), considerando la acción formativa útil, donde se han abordado temas apropiados, con una planificación adecuada de los mismos.

En términos globales, a pesar de las numerosas limitaciones contextuales y técnicas, habituales en la evaluación de programas de intervención educativa (Anguera, Chacón \& Blanco, 2008), el proyecto extracurricular ha sido evaluado positivamente por los/as participantes, con una valoración media de 8,30 sobre una puntuación máxima de 10. Así parece corroborarse cuando se comparan los datos sobre absentismo recabados antes y después de su implementación, en los que se pueden apreciar cierta mejoría en algunos ítems, y cambios notables en el ítem 1 -problemas- y en el ítem 8 -aburrimiento- (figura 4).

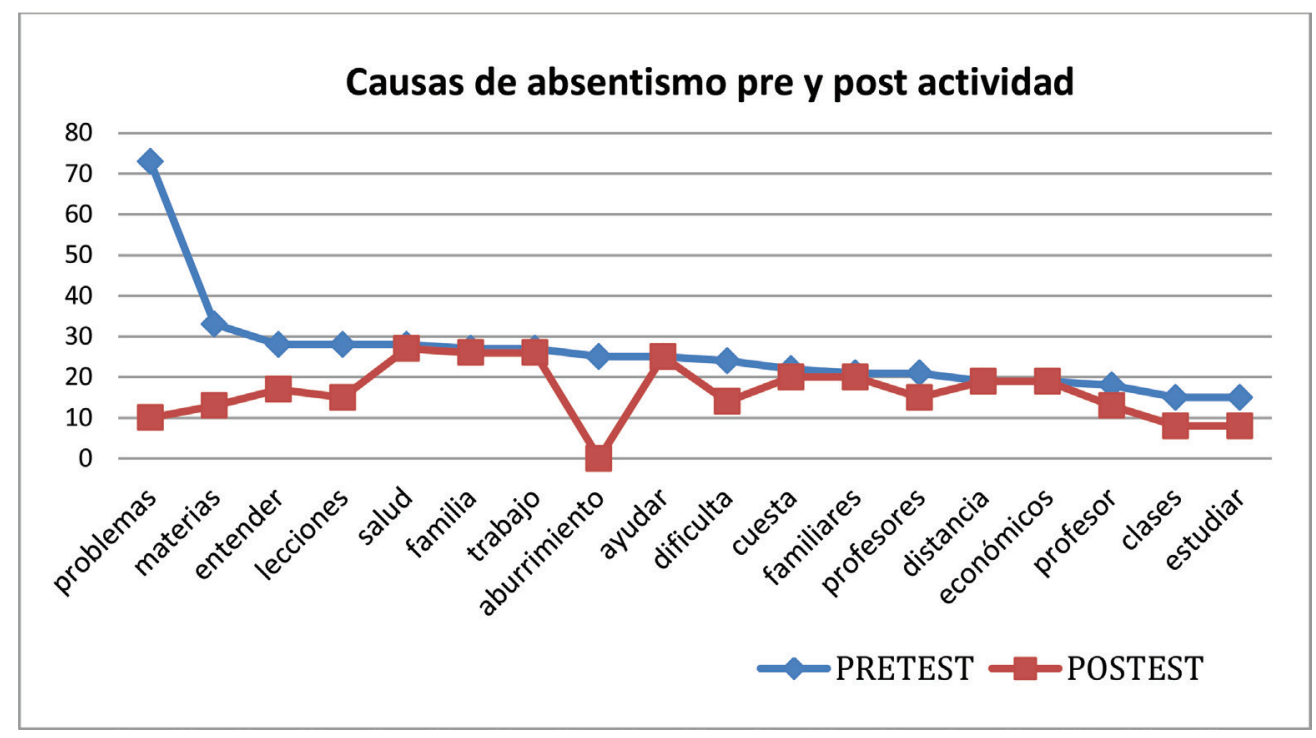

Figura 4 - Causas del absentismo pre y post actividad

Finalmente, al análisis cualitativo de las respuestas a las preguntas abiertas que incorporaba el cuestionario aportó un complemento necesario para comprender mejor ciertos aspectos relevantes de la experiencia del alumnado. En las figuras 5 y 6 se

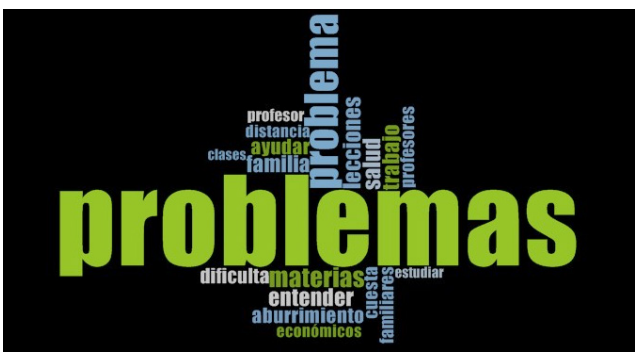

Figura 5 - Nube de palabras factores de riesgo para el abandono escolar o absentismo 
observan las nubes de palabras obtenidas mediante el programa informático NVIVO 11, a partir del análisis de frecuencias de las opiniones expresadas por los participantes. Aparecen las palabras más representativas o con mayor carga porcentual (Costa , Loureiro, \& Reis,2010).

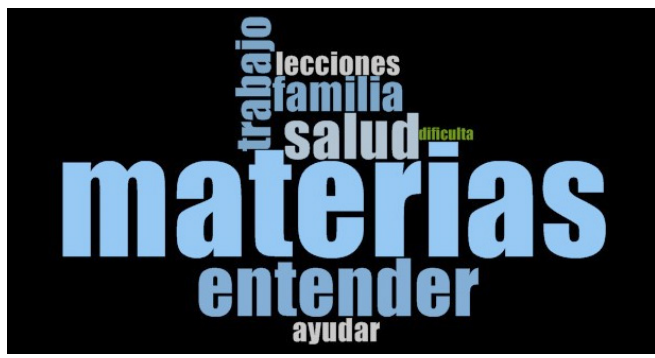

Figura 6 - Nube de palabras factores de protección para el abandono escolar o absentismo

El análisis de contenido de los cuestionarios, combinado con un periodo de observación participante llevada a cabo por una de las investigadoras, avalan la presencia de los factores de riesgo anteriormente mencionados en la población de estudio, con todas las reservas propias de la muestra limitada con la que se ha trabajado. Se ha podido constatar entre los factores del entorno, que la mayoría de los participantes viven en áreas rurales, en algunos casos en zonas económicamente muy deprimidas, donde a las barreras ya mencionadas se añade el tráfico de droga como factor de riesgo importante, ya que en estos contextos resulta un aliciente para la contribución económica a la unidad familiar y por tanto, un motivo para abandonar los estudios. Estudios cuya continuación en muchos casos dependerá más de factores socio-familiares que de la propia motivación del alumnado.

Otros factores como la distancia desde el núcleo familiar al centro, y los costes derivados de ésta (gastos de transporte, problemas en las infraestructuras, etc.) contribuyen negativamente; como comenta un participante: "aveses cuesta que el transporte llegue a buscarnos".

Otros factores más relacionados con el propio centro educativo, como la precariedad laboral del profesorado en alguno de los centros (RC), impide la puesta en marcha y sobre todo la continuidad en la implementación de proyectos educativos que necesitan de un cierto recorrido para aportar resultados relevantes y perceptibles por la comunidad. Todo ello repercute en la experiencia del alumnado, reflejada en comentarios como: "el que nome resulta nada interesante", "hay materias que se me dificultan entenderlas", "son muy rapidos al explicar", "Porque algunas lecciones son aburridas a causa que solo se escribe y no se asen actividades", "no hay dinamica hay aburrimiento", "por los profesores solo hablan y hablan y dan sueño".

De entre los problemas familiares, una de las razones principales por las que no asisten a clase, es la necesidad de ayudar en casa por problemas económicos. Relacionado con lo comentado anteriormente, este indicador de absentismo se aprecia en los siguientes comentarios: "por que aveses el dinero no alcansa para comprar algunos utiles del 
colegio", "Por que el dinero que gana mi mama no puede cubrir todos los gastos escolares", "por que mi padre esta enfermo y no gana mucho", "por que algunos tiene que viajar en bus y no siempre hay dinero y en el colegio no hay bus", "por que no tenemos plata para venir todos los diasnal cole”.

Otras barreras o dificultades provenientes del sistema familiar atañen al plano relacional, como "problemas de conprension departe de mi papa no me llevo ,muy bien con el siempre discutimos", falta de apoyo departe de mi papa nunca me apoya siempre discuto con el no me comprende", "tal vez me hechen de la casa". Y más allá del aspecto puramente relacional, la cuestión pragmática de "traer dinero a casa”, ya sea ayudando en el trabajo a sus progenitores o asumiendo las tareas del hogar y cuidado de familiares: "mi abuela esta enferma y ay que cuidarla", "avese tengo que q cuidar amis sobrinos y no puedo asistir al cole", "si xq aveses tego q trabajar para ayudar a mi mama”, "solo vivo con mi papa y tios me toca velar por mi papa y hacer todo en casa". Y en suma, el bajo nivel socioeducativo de las familias (véase tabla 3), la baja motivación para promover la asistencia a la escuela, ya que la educación no se considera un elemento prioritario para el desarrollo o mejora de la condiciones de vida, constituyen un factor de riesgo transversal.

Finalmente, en lo que concierne a factores de riesgo más directamente relacionados con el/la propio/a alumno/a, algunos manifiestan ser conscientes de sus dificultades de aprendizaje, y de algunos elementos que podrían estar relacionados con éstas, en su mayoría en el plano psicológico expresándolo de la siguiente manera: "por mi problema de aprendizaje", "por que me cuesta desarrollarme o involucrarme en las lecciones", "embeses por falta de concentración", "sufro me agotamiento mental no me concentro con facilidad”. Sí es reseñable la presencia en varios/as participantes con problemas de asma, aunque en general manifiestan tener pocos problemas de salud, y en todo caso ésta no parece ser desde su punto de vista el principal tipo de barrera para su continuación en los estudios.

\section{Conclusiones}

Los resultados de aprendizaje alcanzados por los estudiantes satisfacen los objetivos del programa formativo. La tasa de rendimiento y de éxito del programa ha sido muy elevado. De igual modo, los resultados de las actividades multimedia, en las que han participado los centros colaboradores, han sido notablemente satisfactorios. Por lo tanto, hay que señalar que no se han detectado anomalías o evidencias significativas en torno a la evaluación, para el que cada uno de los investigadores ha dispuesto el correspondiente sistema de pruebas. A partir de aquí, se sugiere la posibilidad de cambiar el volumen de materias, algunas resultan muy tediosas, incluso se propone la posibilidad de motivar al alumnado a través de actividades prácticas, como las que se han llevado a cabo con el proyecto, dado el éxito y grado de satisfacción que los alumnos han manifestado. Por otra parte, se considera importante estar alerta sobre la población de estudiantes con capacidades diferentes para facilitarles las medidas específicas que puedan ser necesarias para proporcionarle una compensación educativa de forma personalizada, dirigida a la atención integral. Otro aspecto que puede tenerse en cuenta, como propone el Gobierno Vasco (2003) es discriminar positivamente, en la asignación de recursos, a aquellos Centros que atienden en mayor grado a estudiantes con riesgo de exclusión social. Así como establecer protocolos de actuación ante la falta de asistencia a clase del alumnado. 
Entre las quejas de los estudiantes muchas de ellas se centraron en la demanda de un mayor equilibrio en la asignación de tareas, deberes y trabajos de clase. En segundo lugar, vemos cómo se producen diferencias relevantes en el abandono de estudios, entre diferentes sectores de la población. Las tasas de abandono más elevadas se dan en los estudiantes con bajo sentimiento de pertenencia a la comunidad.

Más allá de los datos cuantitativos obtenidos, y del impacto proximal de las actividades, la experiencia del alumnado con el proyecto extracurricular ha supuesto una apertura a la comunidad, ya que para muchos de ellos/as su participación ha supuesto, entre otros, salir de su círculo más inmediato por primera vez e interaccionar con alumnado y profesorado de otros centros. En este sentido, el impulso motivacional que haya podido generar este tipo de actividades puede haber contribuido a una reflexión más profunda sobre sus propias capacidades y posibilidades, y con ello una nueva perspectiva en su percepción del futuro académico y personal. Asimismo, el diseño de las actividades ha incluido una experiencia intergeneracional, y con ello la potenciación del sentimiento de pertenencia a la comunidad. Este contacto con los mayores de su comunidad, aunque no ha sido considerado en la evaluación, probablemente ha aportado beneficios no directamente tangibles pero en todo caso bidireccionales: al alumnado le ha permitido conocer y participar más a fondo de su medio social, y con ello facilitar una mejor integración de las personas mayores en sus experiencias vitales; y por parte de estas últimas, un posible efecto distal sería cierto aumento en su autoestima, al tener la oportunidad de percibir que aún siguen siendo útiles a las nuevas generaciones, más allá del beneficio que por sí mismo supone el sentirse miembros activos de la sociedad.

Por otra parte, la evaluación del proceso y los resultados del proyecto refuerza la idea de que ante fenómenos de alta complejidad, como en el caso del absentismo y abandono escolar, donde son múltiples los factores de riesgo (si bien también los posibles factores de protección), la respuesta de la sociedad requiere de una intervención multidisciplinar. No se trata de un problema personal, familiar, ni tan siquiera exclusivamente "educativo", se trata sobre todo de un problema social, donde no resultan suficientes las intervenciones aisladas y parciales que las distintas Administraciones pueden poner en marcha ante los casos detectados, las cuales, si bien son imprescindibles, no parecen alcanzar los complejos mecanismos subyacentes. En consecuencia, se requiere de una perspectiva más amplia, de un abordaje integral, basado en políticas activas y proactivas, no únicamente paliativas. Y se hace, por tanto, necesaria una coordinación interdisciplinar de las diferentes instituciones implicadas, dirigida a prevenir, reducir y, en su caso, solventar las dificultades y barreras a fin de minimizar en la medida de lo posible la probabilidad de deserción escolar.

\section{Referencias}

Abreu, A., Rocha, Á., de Carvalho, J. V., \& Cota, M. P. (2017). The electronic booklet on teaching-learning process: Teacher vision and parents of students in primary and secondary education. Telematics and Informatics, 34(6), 861-877.

Anguera, M.T., Chacón, S., \& Blanco, A. (2008). Evaluación de programas sociales y sanitarios. Madrid: Síntesis 
Arguedas, I. (2011). Acceso a la permanencia en la educación secundaria en estudiantes costaricenses. Revista de Educación y Desarrollo, 19, 29-34.

Arguedas, I., \& Jiménez, F. (2007). Factores que promueven la permanencia de estudiantes en la Educación Secundaria. Actualidades Investigativas en Educación, $7(3), 1-36$.

Costa, A. P., Loureiro, M. J., \& Reis, L. P. (2010). Metodologia Híbrida de Desenvolvimento Centrado no Utilizador aplicada ao Software Educativo. RISTI-Revista Ibérica de Sistemas e Tecnologias de Informação, (6), 1-16.

Gobierno Vasco. (2003). Programa para Garantizar el Derecho a la Educación: erradicación de la desescolarización y el absentismo escolar en el territorio de Biskaia. Gobierno Vasco.

Gómez, M., Vicario, B. P., García, C. L., \& García, A. S. (2014). Percepciones de los empresarios de Pymes rurales sobre la integración de las TIC. RISTI-Revista Ibérica de Sistemas e Tecnologias de Informação, (SPE2), 71-84.

Goodenow, C. (1993). The psychological sense of school membership among adolescents: Scale development and educational correlates. Psychology in the Schools, 30(1), 79-90.

Jiménez, W., \& Gaete, M. (2010). Informe de Investigación: Abandono (deserción) escolar en la enseñanza secundaria en Costa Rica 2009-2010. San José, Costa Rica: Ministerio de Educación.

Martín-Cilleros, M., Sánchez-Prada, A., Delgado-Álvarez, M. C., Sánchez-Gómez, M. C., (2017). Evaluación multimétodo de un proyecto extracurricular tecnológico para prevenir la deserción escolar, In CIAIQ 2017, Congresso Iberoamericano em Investigação Qualitativa, Investigação Qualitativa em Engenharia e Tecnologia, Volume 4, pp. 75-84, Salamanca, Spain

Ragin, C. C. (2014). The comparative method: Moving beyond qualitative and quantitative strategies. Oakland, CA: Univ of California Press.

Ros, I. (2014). La cooperativa de trabajo asociado de trabajadores y el sentimiento de pertenencia de su profesorado. Qurriculum: Revista de teoría, investigación y práctica educativa, (27), 85-104.

Sánchez-Gómez, M. C., \& Martín-Cilleros, M. V. (2017). Implementation of focus group in health research. In Computer Supported Qualitative Research (pp. 49-61). Cham: Springer International Publishing. 